\title{
Process Control System Design Based on Genetic Algorithm
}

\author{
Ping Liu, Yongchao Xie, Xiaoqing Ding and Mingwei Wang \\ Xiamen University Tan Kah Kee College,Zhangzhou,Fujian 363105,China
}

Key words: Process control; Genetic algorithm; Kingview; Double-tank

Abstract: Because the process control laboratory supporting software only provides the conventional PID algorithm, the paper combines with advanced control algorithm---genetic algorithm to design process control experiment. This paper emphasizes on using kingview language to realize the selection and reproduction, crossover and mutation process of genetic algorithm. At the same time, this article introduces the method which how to generate the tank characteristic curve, the valve characteristic curve and the experiment report. We can conclude that the design of experiments is effective and have great help for the students to grasp the genetic algorithm from the feedback of the students and the experiment results.

\section{Introduction}

Process control laboratory are generally equipped with kingview software, but the algorithm provided by vendors is usually only conventional PID algorithm. So, the students can only learn advanced algorithm theories in class and unable to verify in the laboratory, which makes them hard to master advanced algorithm .In addition, the function of the software proided by vendors is single:generally contain only PID parameter settings, hand/automatic switch,real-time/history curve, and don't have dynamic valve features .Faced with the problems above, it is necessary to develop advanced multi-function experiment platform. Take the genetic algorithm as an example, the paper introduce the design process of the double water tank liquid level control system.

\section{Design scheme}

In double water tank liquid level control system, the function of the software include: liquid level single loop control, pressure single loop control, hand / automatic switch, PID parameter setting, historical curve, real-time curve, exit experiment and so on. The design makes some changes on the basis of the original function: increase the genetic algorithm, valve characteristic, the characteristic of the water tank, the experimental report; decrease the PID parameter settings module of liquid level single loop. The main page of the final design of the experiment is as shown in figure 1.Liquid level single loop control module uses the genetic algorithm, The function block diagram of the genetic algorithm module is shown in figure 2.It consists of four sub modules: genetic algorithm parameter settings, manual/automatic switch panel, parameters optimization curve and history/realtime response curve.

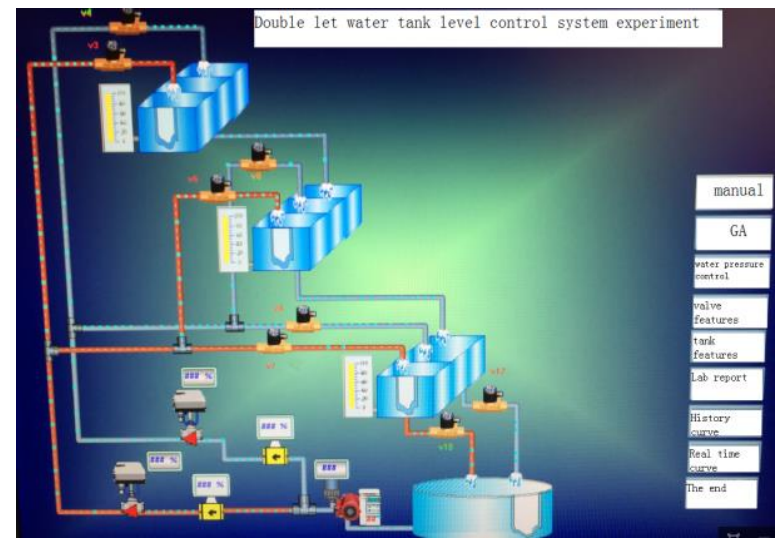

Figure 1. The main page of double water tank liquid level control system based on genetic 
algorithm

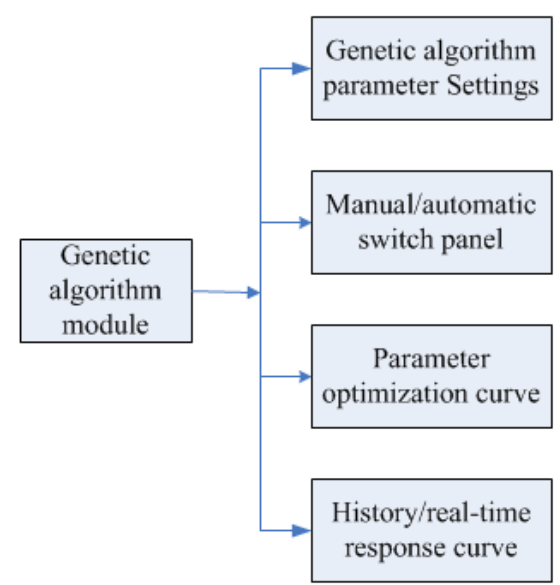

Figure 2. The function block diagram of the genetic algorithm

\section{The realization of the $G A$}

Genetic algorithm(GA) is an overall random search method, it contains five steps: encoding, generation of the initial population, adaptive evaluation, selection and reproduction, crossover and mutation. The block diagram of liquid level single loop control is shown in figure 3. In figure 3, broad block represents the control module of the system. Its input signals is the real-time deviation, its algorithm is the genetic algorithm, its function is to find the optimal $\mathrm{KP}, \mathrm{Ki}, \mathrm{kd}$.

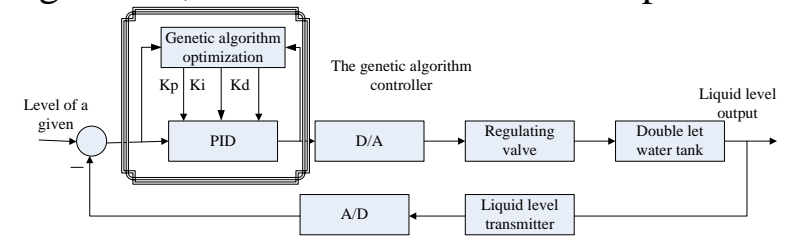

Figure 3. The block diagram of liquid level single loop control

\subsection{The part of MATLAB implementation}

Due to the limitation of the Kingview programming, The generation of initial population and coding are done by matlab. You can refer to reference 1 to understand the process of concrete implementation. When you using MATLAB programming, it is necessary to establish the mathematical models of the double tank, the valve and the liquid level transmitter, and then calculate the mathematical model of the generalized controlled object.

\subsubsection{Coding}

The so-called encoding is a process to represent data into genotype string structure before the search. Simulate the process, the corresponding MATLAB codes with $\mathrm{Kp}, \mathrm{Ki}, \mathrm{Kd}$ coding are as follows(liu,2004)

$\operatorname{MinX}(1)=\operatorname{zeros}(1)$;

$\operatorname{MaxX}(1)=50 *$ ones(1); \% The Kp range

$\operatorname{MinX}(2)=\operatorname{zeros}(1)$

$\operatorname{MaxX}(2)=1.0 *$ ones(1); \% The Ki range

$\operatorname{MinX}(3)=\operatorname{zeros}(1)$

$\operatorname{MaxX}(3)=1.0 *$ ones( 1$) ; \quad \%$ The Kd range

$\mathrm{E}=\operatorname{round}(\operatorname{rand}(40,3 * 10)) ; \% \mathrm{Kp}, \mathrm{Ki}, \mathrm{Kd}$ coding 


\subsubsection{Initial population generation, taking the Kp parameter as an example}

In the implementation of parameter optimization, the individual using the decimal encoding. The matlab codes are as follows:

for $s=1: 1: 40$

$\mathrm{m}=\mathrm{E}(\mathrm{s},:)$;

$\%$ select individual

$\mathrm{y} 1=0 ; \mathrm{y} 2=0 ; \mathrm{y} 3=0$

$\mathrm{m} 1=\mathrm{m}(1: 1: 10)$;

\%select the Kp parameters

for $i=1: 1: 10$

$\mathrm{y} 1=\mathrm{y} 1+\mathrm{m} 1(\mathrm{i}) * 2^{\wedge}(\mathrm{i}-1) ; \quad \%$ Convert to decimal

end

$\operatorname{Kpid}(\mathrm{s}, 1)=(\operatorname{Max} X(1)-\operatorname{MinX}(1))^{*} \mathrm{y} 1 / 1023+\operatorname{MinX}(1)$;

Initial population of kingview programming will uses $\mathrm{Kp}, \mathrm{Ki}, \mathrm{Kd}$ (40 individuals)which are obtained by MATLAB simulation.

\subsection{Realization of Kingview.}

Kingview programming language and $\mathrm{C}$ language are roughly similar, but it is not as flexible as $\mathrm{C}$ language, Such as: no statement like for cycle. Therefore, it is not convenient to use genetic algorithm to realize the evolution of $\mathrm{N}$ generation. For this reason, the ideas of genetic algorithm programming need some adjustments. In order to achieve the equivalent evolution of 40 generations, 40 individuals should be picked out simultaneously to participate in genetic operation. Program flow chart is shown in figure 4.

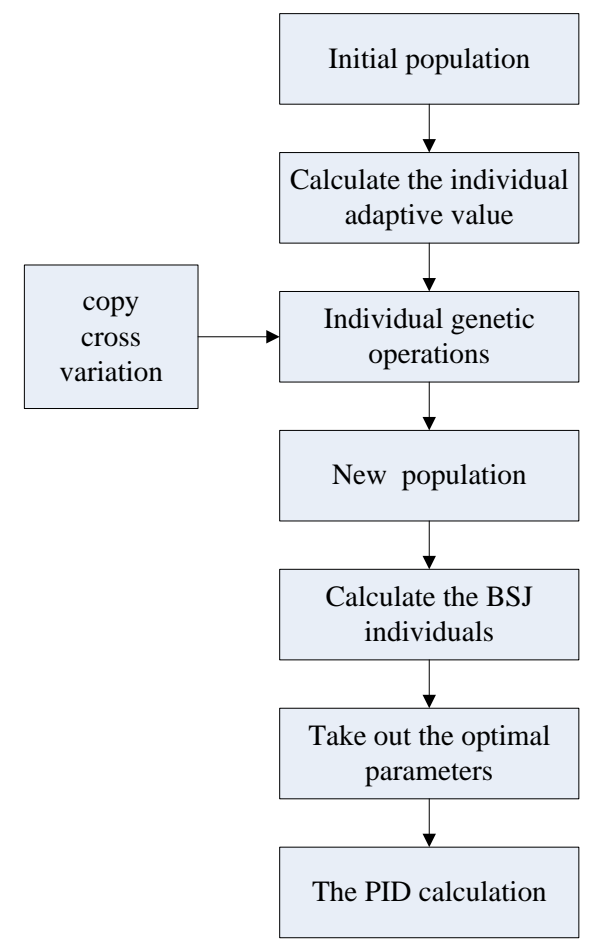

Figure4. The genetic algorithm flow based on Kingview.

\subsubsection{Calculate fitness function.}

Define the error of given value and the measured value as the optimization index BSJ, take its reciprocal as adaptability evaluation function fi.Through computation of fi, we can get each individual adaptive value. 


\subsubsection{Selection and reproduction}

In the genetic algorithm, the adaptable individuals have a high probability to give birth to one or more offspring. In programming processing, according the size of the fi, we determine which individual is selected and copied. The Kingview programming are as follows:(Ozpineci,2005; Chang,2002; Wang,2014; Yu,2009)

if(fi>a)

$\{\mathrm{TE} 11=\mathrm{Kp} ; \mathrm{TE} 12=\mathrm{Kd} ; \mathrm{TE} 13=\mathrm{Ki} ;\}$

else

$\{$ TE11=BestS1;TE12=BestS2;TE13=BestS3; $\}$

Define a variable a, it is available for users to set and debug.TE1i are the intermediate variable to store the optimal individual. BestSi are the best $\mathrm{Kp}, \mathrm{Ki}$ and $\mathrm{Kd}$ parameters in the MATLAB simulation.

\subsubsection{Crossover}

In the genetic algorithm, a new generation of individual can be obtained through the crossover operation. The new generation not only embodies the characteristics of their parents, but also reflects the characteristics of the information exchange. Kingview programming are as follows:

float rand $0=$ random(seed);//Write random function

float $\operatorname{rand} 1=\operatorname{random}(\operatorname{rand} 0)$;

float rand2=random(rand1);//Generate random number

temp=rand(rand1);

if(Pc>temp)

$\{b=\operatorname{rand}(\operatorname{rand} 2)$;

TempE11=b*TempE21+(1-b)*TempE11;

TempE12=b*TempE22+(1-b)*TempE12;

TempE13=b*TempE23+(1-b)*TempE13;\}//Individual crossover

The Pc is defined as memory variables.Program segment reflects the cross of two individuals, and manifests the randomness, which conforms to the meaning of the cross in the genetic algorithm.

\subsubsection{Variation}

Variation provides the opportunity for a new generation of individual. According to the property of variation, the corresponding Kingview procedures of $\mathrm{Kp}$ are as follows(Lin,2009)

if $($ Pm1 $1>$ Pm_rand11)

TempE11=Mean1+Dif1*rand(rand1)-Dif1*0.5;

Variables Pm1, Mean1, Dif1 are defined as memory variables. Pm_rand11, rand(rand1) are the random constants.

40 individuals of the initial population have experienced the process of selection, reproduction, crossover and variation. Then, they evolved into 40 new individuals.Calculate the BSJ of individual respectively, formulate the minimum function, and select the minimum $\mathrm{BSJ}$, corresponding $\mathrm{Kp}, \mathrm{Ki}$, $\mathrm{Kd}$ as results of parameter optimization. Next, kingview program use the $\mathrm{Kp}, \mathrm{Ki}, \mathrm{Kd}$ to complete the design of genetic algorithm controller.

\section{The realization of the characteristic test}

In order to allow users to have a more intuitive understanding of modeling and the device features, the system are added water tank and valve characteristics. They have the same realization method: as long as set variables in $\mathrm{X}-\mathrm{y}$ axis curve. Take the water tank features module as an example, $\mathrm{X}, \mathrm{Y}$ are respectively defined as the variables of water inflow and level. According to the characteristic curve, it can be seen clearly that the liquid level changes with the water inflow, which can make it possible to adjust the outlet opening appropriately to achieve a better control. 


\section{The generation of the experimental report}

Automatic generation of experimental reports can not only reduce the workload of users, but also can record the data effectively.Experimental report design as shown in graph 5.It can quickly achieve by using Kingview reporting tools.

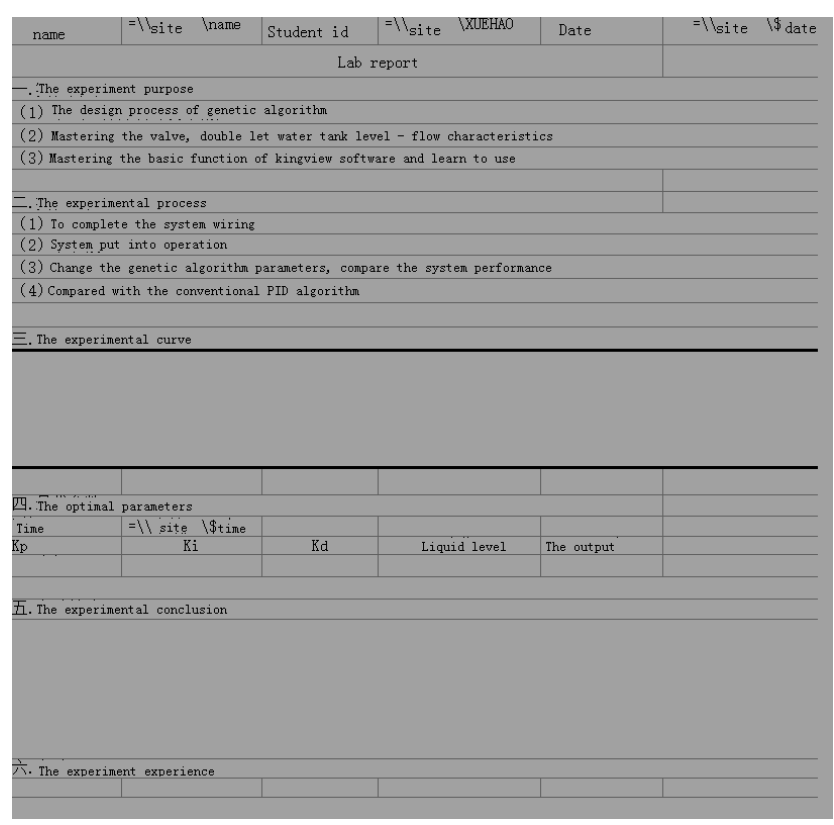

Figure 5. Experimental report.

\section{The experimental results}

The experimental equipment is shown in figure 6.Before the experiment, it is required to inspect the water level of water tank, and open the manual valve in the corresponding pipe.After the completion of the on-site equipment debugging, it is required to complete circuits connection of liquid level single loop and pressure single loop.

After completion of external hardware and wiring, open the experiment project file., Then, the operation in accordance with the following order.

(1) Set up corresponding genetic algorithm parameters in Kingview interface: $a=0.2, P c=0.7$, Pm1=0.199750, Mean1=0.5, Dif1=30;

(2)Open the electric adjustable valve manually;

(3)Pressure single loop can be directly led into the closed loop operation;

(4) Put liquid level single loop into the closed loop operation when liquid level almost reaches the given value $(10 \%)$.

It can be seen from the debug results, the system can quickly optimize the parameters, and the control performance is good. In order to verify whether the system has a strong robustness, the given value is increased by 35 to 40 when the system is stable. The experimental results are shown in Figure 7. From the figure 7, the stability of the system is very good with zero overshoot, adjustment time is very short,steady-state error is almost zero. 


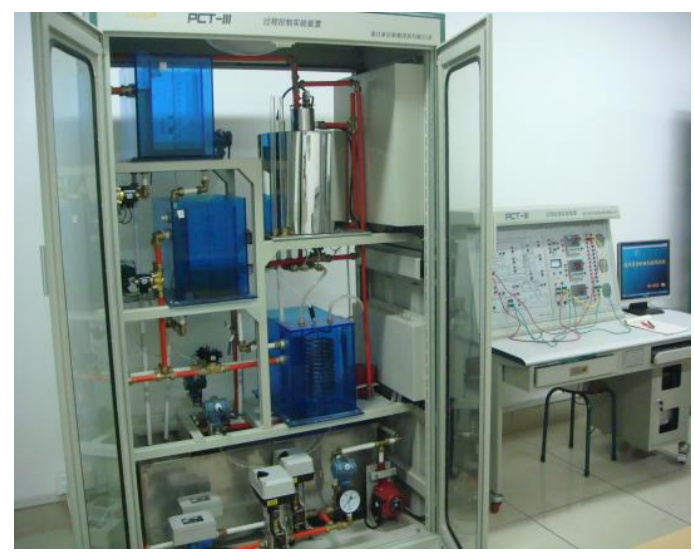

Figure6. The experimental equipment

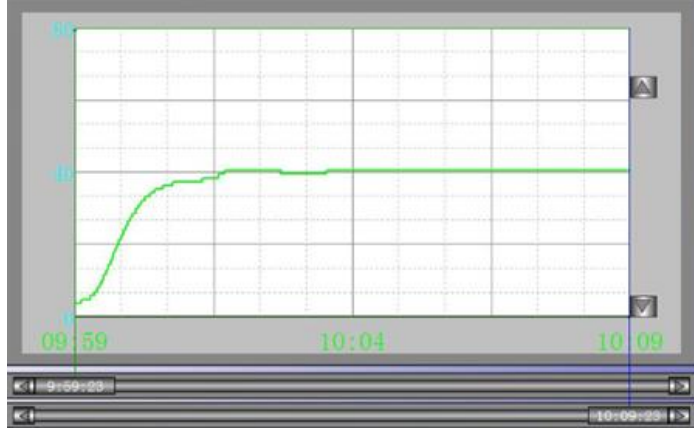

Figure 7. Liquid level time domain response curve

\section{Conclusion}

Analyse genetic algorithm using kingview programming, it fully embodies the steps of genetic algorithm, so the algorithm is conform to the requirements.From the results, the system can automatically complete parameters optimization, it has a strong robustness.Compared with the conventional PID control ,it has a better stability and faster response ability.Developing such as advanced algorithm experimental system, it not only make up for the shortage of the configuration software provided by manufacturers, but also important to help students understand and master the advanced algorithm of the control system.

\section{References}

[1] Jin-kun liu. The advanced PID control MATLAB simulation [M]. Beijing: electronic industry press, 2004.

[2] B.Ozpineci, L.M. Tolbert, and J. N. Chiasson, Harmonic optimization of multilevel converters using genetic algorithms, IEEE Power Electronics Letters, Vol. 3,No. 3, pp.92-95, Sep. 2005.

[3] ChangliangLiu,YaoWanye,Zhai Yongjie,etal.Animproved genetic algorithm and its application in thermal

[4] Process control [ J] . Process Automation Instrumentation,

[5] 2002, 23 (9): 13 -16.

[6] Jian Wang, Yongchang Song. Kingview software introduction and typical application [M]. Beijing: China power press, 2014.

[7] Chengbo Yu, Lian Zhang, Xiaoqian Hu. Automatic control priciple [M]. Beijing: tsinghua university press, 2009.

[8] Jinguo Lin, Li Zhang, Lijuan Li. Process control [M]. Nanjing: southeast university press, 2009.

[9] Guangnan Xuan, Runwei Cheng. Optimization of genetic algorithm and engineering [M]. Beijing: tsinghua university press, 2004. 